\title{
Introducing New Priority Setting and Resource Allocation Processes in a Canadian Healthcare Organization: A Case Study Analysis Informed by Multiple Streams Theory
}

\author{
Neale Smith ${ }^{1 *}$, Craig Mitton ${ }^{1,2}$, Laura Dowling ${ }^{3}$, Mary-Ann Hiltz ${ }^{4}$, Matthew Campbell ${ }^{4}$, Shashi Ashok Gujar ${ }^{4,5}$
}

\begin{abstract}
Background: In this article, we analyze one case instance of how proposals for change to the priority setting and resource allocation (PSRA) processes at a Canadian healthcare institution reached the decision agenda of the organization's senior leadership. We adopt key concepts from an established policy studies framework Kingdon's multiple streams theory - to inform our analysis.

Methods: Twenty-six individual interviews were conducted at the IWK Health Centre in Halifax, NS, Canada. Participants were asked to reflect upon the reasons leading up to the implementation of a formal priority setting process - Program Budgeting and Marginal Analysis (PBMA) - in the 2012/2013 fiscal year. Responses were analyzed qualitatively using Kingdon's model as a template.

Results: The introduction of PBMA can be understood as the opening of a policy window. A problem stream defined as lack of broad engagement and information sharing across service lines in past practice - converged with a known policy solution, PBMA, which addressed the identified problems and was perceived as easy to use and with an evidence-base from past applications across Canada and elsewhere. Conditions in the political realm allowed for this intervention to proceed, but also constrained its potential outcomes.

Conclusion: Understanding in a theoretically-informed way how change occurs in healthcare management practices can provide useful lessons to researchers and decision-makers whose aim is to help health systems achieve the most effective use of available financial resources.

Keywords: Priority Setting, Resource Allocation, Multiple Streams Theory, Tertiary Care Health Centre, Canada Copyright: $\odot 2016$ by Kerman University of Medical Sciences

Citation: Smith N, Mitton C, Dowling L, Hiltz MA, Campbell M, Gujar SA. Introducing new priority setting and resource allocation processes in a Canadian healthcare organization: a case study analysis informed by multiple streams theory. Int J Health Policy Manag. 2016;5(1):23-31. doi:10.15171/ijhpm.2015.169
\end{abstract}

Article History:

Received: 11 June 2015

Accepted: 14 September 2015

ePublished: 24 September 2015
*Correspondence to:

Neale Smith

Email: neale.smith@ubc.ca

\section{Key Messages}

Implications for policy makers

- Introducing new formal processes for priority setting and resource allocation (PSRA) into healthcare organizations is a challenge of change management.

- Concepts from the policy sciences, such as John Kingdon's Multiple Streams theory, can help policy-makers understand and assess the alignment of factors which present the greatest windows of opportunity to introduce change.

- Policy-makers should ensure that proposed policy actions fit with agreed problem definitions, and action should be feasible within the context of political constraints.

Implications for public

Healthcare organizations can make more effective and efficient use of their limited financial resources, and produce better outcomes for patients and the public, if they use established methods and tools to help make choices about what programs and services to fund. This research should help decision-makers in healthcare organizations to figure out what conditions will help, or hinder, them in moving forward with implementing these changes.

\section{Background}

There is now an established and growing body of literature on priority setting and resource allocation (PSRA) in healthcare. This literature articulates - normatively and empirically - the case for formalized processes. Key features of good formal processes have been outlined, and facilitators and barriers to good practice enumerated. However, we know remarkably little about why and how healthcare organizations reach the decision to restructure their PSRA practice in the first place. Few studies have looked at introduction of formal PSRA methods in hospitals (compared to assessment of the application of such methods) - only $8 \%$, or 2 out of 24 , published papers, according to a recent review. ${ }^{1}$ This is a gap in the literature. From a research perspective, this study will 
tell us more about what factors serve to spur decision-maker commitment to changing PSRA processes. Practically, it will get decision-makers thinking about these conditions - there would be great value in enabling them to judge when the timing is not right for attempting to implement new formal PSRA processes.

In this paper, we analyze one case instance of what put the need to do resource allocation differently onto the decision agenda of a Canadian healthcare organization. The organization, the IWK Health Centre in Halifax, NS, Canada, chose to implement the priority setting process known as Program Budgeting and Marginal Analysis, or PBMA. (Note that the actual evaluation of the new system is not the primary focus of this report.) Smith et $\mathrm{al}^{2}$ have argued that despite their clear relevance, public policy analysis frameworks and concepts have rarely been employed by scholars in this field. We thought that there would be value in applying such a lens to this case; in particular, the concepts of Kingdon's multiple streams theory seemed likely to give us purchase upon key issues that could enable understanding and learning from this case. Multiple streams is one of the more commonly used models in policy studies and is argued to be among those with the greatest analytical heft. ${ }^{3,4}$

Multiple streams is a theory of agenda-setting, ${ }^{5,6}$ that is, a means for explaining how different issues do or do not become raised to a level where decision-makers feel it is necessary to discuss concerns, debate alternatives, and adopt explicit policy choices. Ridde suggests that it is an "indispensable" tool for understanding these processes. ${ }^{7}$ Briefly, multiple streams argues that policy change occurs when a 'policy window' is opened by the joining of a problem stream (an issue), a policy stream (a solution) and a politics stream (driven by public opinion and/or partisan electoral activities). The streams develop independently and it typically takes a 'policy entrepreneur' to join them and open the window for change. Such entrepreneurs can be politicians, administrators, or even advocates or researchers external to the decision-making system but who have substantial credibility and influence within it.

Kingdon's model was developed originally to apply to national-level transportation and health policy-making in the US Congress; it has however subsequently been applied to health policy in a range of other national contexts, both in developed ${ }^{8,9}$ and developing countries. ${ }^{7}$ It has also been successfully used at the subnational level, through studies of policy action in both US states ${ }^{10,11}$ and Canadian provinces. ${ }^{12-14}$ Importantly, it has been applied to study the introduction of new decision-making practices-eg, Health Impact Assessment ${ }^{15}$ - which is the closest parallel and precedent for how we employ it in the current research. Tenbensel has noted that multiple streams theory is subject to 'conceptual stretch, being applied to situations beyond those originally theorized. ${ }^{16}$ Here, we are using the core concepts from the theory as an organizing framework or template to help practitioners understand their experience, not necessarily to lead to expansion or refinement of the theory itself.

The context for this case study is the IWK Health Centre in Halifax, NS, Canada. The IWK is a tertiary care facility serving children and women from across Canada's Maritime provinces. Provincial government funding pressures placed the organization in a difficult financial situation. In $2011 / 2012$, budget allocations were frozen, which equated to a $7 \%$ real reduction in funding at the IWK when inflation is factored in. The Executive Leadership Team (ELT) undertook several measures at that time to cut spending and maintain budget balance. This process was criticized internally for failing to fully engage stakeholders or to adopt an integrated, systems view of resource allocation. Managers, physicians, and other health professionals claimed that the strategies chosen to address the budget shortfall were not the ones they would have identified if asked. On top of this, the 2012/2013 fiscal year presented the IWK with a further 3\% reduction in total health transfers from the province. Rather than continue in crisis mode, the ELT determined to get in front of the challenge by transitioning to a new, explicit approach to resource allocation. The PBMA framework was selected: it was expected to assist the organization in managing financial pressures by providing a structured method for identifying and comparing investment and disinvestment options. (Box 1 provides a summary of the PBMA approach.)

Three sections follow. The Methods section describes data collection and analysis procedures. Results are presented in three parts according to the Kingdon model: problem stream, policy stream, and politics stream. Finally, the Discussion and Conclusion section considers the value added by employing this analytic approach, notes implications for PSRA practice

\section{Box 1. Stages in a PBMA Exercise}

1. Determine the aim and scope of the priority setting exercise

- Decide whether PBMA will be used to examine changes in services within a single Department or program or between Departments/programs.

2. Compile a "program budget"

- Current resources assigned to each defined program should be identified and quantified.

3. Form a "marginal analysis" advisory panel

- Key stakeholders (managers, clinicians, consumers, etc.) should be able to contribute to the priority setting process through this formal Advisory Panel, or in some other clearly defined manner.

4. Determine locally relevant decision-making criteria

- All proposed investments or disinvestments will be assessed against these criteria, which should reflect the mission and mandate of the organization and the values of the community which it serves.

5. Identify options for (a) Service growth, (b) Resource release from gains in operational efficiency, $(c)$ Resource release from scaling back or ceasing some services

- These proposals can be developed by an organization's senior leaders or solicited from staff through an engagement process.

6. Evaluate investments and disinvestments

- Using the agreed-upon criteria, managers will consider options and make recommendations for moving resources from 5 (b) and 5 (c) to 5 (a) above.

7. Validate results and reallocate resources

- The leadership group, with additional outside input as desired, will assess the allocation decisions reached through the process and make reasoned adjustments, if necessary.

Adapted from Peacock et al. 
in healthcare service delivery organizations, and suggests areas for further future research.

\section{Methods}

This research should be considered as a case study ${ }^{18}$ of practice change in one organization. Data collection involved 26 individual face-to-face interviews. Interviews were chosen as the research method in order to allow each participant to tell his or her own personal story of how they experienced the PBMA process; we sought to obtain narratives with a clear beginning, middle and end. Interviewees were purposively selected based on their known relationship to the PBMA implementation. They included 5 members of the ELT, 14 middle managers (Department Heads, Directors, and Managers), 6 care providers ( 3 senior physicians and 3 allied health workers), and one community member who had been recruited by the IWK to provide a public perspective in PBMA discussions. The full range of child health services provided by the IWK were represented (eg, primary health, mental health and addictions, pediatric medicine, rehabilitation). See Table for more information about participants. Five other individuals declined or were unable to accommodate a request to participate ( 3 middle managers, one physician and one allied health practitioner). Those who declined were not asked to provide their reason for doing so. The final response rate was $84 \%$. All interviews were conducted during fall 2013 and lasted between 30 and 60 minutes. Interviews were conducted by a research associate hired for this project. She had no previous connection to the IWK or to the PBMA process. Interviews were digitally recorded and subsequently transcribed.

Data were analysed against Kingdon's agenda setting framework. That is, we identified and coded statements which appeared to contain respondents' views of:

a. Problem: why PSRA practice at IWK needed to change, or not

b. Policy: what options for doing PSRA differently were considered

c. Politics: what internal or external interest group pressures affected IWK's intent to introduce PBMA and

Table. Interview Participants

\begin{tabular}{|c|c|c|}
\hline Respondent Group & Gender & $\begin{array}{l}\text { Extent of Involvement } \\
\text { with PBMA }\end{array}$ \\
\hline Executive Leadership Team & $\begin{array}{l}3 \mathrm{~F} \\
2 \mathrm{M}\end{array}$ & $\begin{array}{l}\text { High }=3 \\
\text { Medium }=2 \\
\text { Low }=0\end{array}$ \\
\hline Middle management & $\begin{array}{l}9 \mathrm{~F} \\
5 \mathrm{M}\end{array}$ & $\begin{array}{l}\text { High }=2 \\
\text { Medium=6 } \\
\text { Low }=6\end{array}$ \\
\hline Physicians & $\begin{array}{c}1 \mathrm{~F} \\
2 \mathrm{M}\end{array}$ & $\begin{array}{l}\text { High }=0 \\
\text { Medium=2 } \\
\text { Low }=1\end{array}$ \\
\hline $\begin{array}{l}\text { Allied Health Professionals, } \\
\text { Front line workers, public } \\
\text { members }\end{array}$ & $\begin{array}{l}2 \mathrm{~F} \\
2 \mathrm{M}\end{array}$ & $\begin{array}{l}\text { High }=0 \\
\text { Medium=3 } \\
\text { Low }=1\end{array}$ \\
\hline
\end{tabular}

Abbreviation: PBMA, Program Budgeting and Marginal Analysis.

aHigh involvement $=$ a leading or coordinative role in introducing and overseeing PBMA; Medium involvement = participated on a working group or steering committee; assesses proposals for investment or disinvestment; Limited involvement $=$ prepared and submitted proposals to the PBMA process. the unfolding of this change effort.

We consider this coding of statements into categories of an established analytical framework to be an application of template analysis method. ${ }^{19}$

Two of the authors (NS and LD) collaborated to produce the preliminary results. These were reviewed by the research team as a whole, which included 2 academic researchers from Western Canada, the onsite research associate, and four current or former IWK staff members with direct knowledge of the PBMA process, including a member of the IWK's executive leadership. The purpose of the review was to provide additional input to identify weaknesses in the emerging arguments, or insider information that would help clarify the context of respondents' comments. Themes were refined in this way, but no substantial additions or alterations resulted.

This project received formal ethics approval at both the University of British Columbia, and the IWK.

\section{Results}

\section{A. Problem Stream}

Existing PSRA processes are perceived to have been inadequate. Two main themes in the interviews reflect participants' dominant understanding of the nature of the PSRA problem at the IWK: lack of broad engagement, and a 'silo'd' allocation structure.

The problem is repeatedly described as lack of broad engagement by staff with PSRA: "big problem with it was as much as we tried to involve the front line folks, you know, directors, managers and others, they really didn't feel engaged. They didn't feel involved." (\#1: ELT). "Prior to PBMA there was a lot of discontent across the organization that basically the process was limited to a few people" (\#5: ELT) - physicians and mid-level managers were among those feeling disenfranchised. "It was pretty top down and people were getting a bit disengaged and cynical.” (\#22: Manager or other participant, working group member).

Lack of engagement leads to a number of implementation problems. There is, for instance, doubt as to whether or not staff have the understanding and buy-in needed to accept resource reallocation when it affects them. "[We need] a process that engages people as much as possible in those decisions, so that while they may end up not totally agreeing with decisions, they have the context, they understand the realities of what our environment is, and they are participants in the trade-off process." (\#2: ELT).

Any new PSRA process would be judged by its ability to effectively "get different expertise around the table." (\#2: ELT).

"PBMA was introduced as a strategic initiative at the organization. So an opportunity for everyone in the organization to have their say on and provide their expert opinion on how we could do things differently.... We encouraged and provided mechanisms for those ideas to be brought forward in a way that didn't occur in the past" (\#10: Manager or other participant).

"PBMA to me represented a fairly robust attempt by the hospital to actually find out what the workers have got there and to get the good ideas that had actually been suppressed." (\#22: Manager or other participant, working group member). "The part that was particularly good was that the decision- 
making as to how we would reassign resources was made by front line workers because they're the ones that know." (\#3: ELT).

The second element of the prevailing problem definition is that the previous model of priority setting failed to link resource allocation to overarching organization goals, eg, as articulated in the strategic plan. Before, things were done in silos. "My understanding was that there was no general centre-wide approach to doing it [before]. It was more ... silo approach." (\#29: Manager or other participant, working group member). There was no "sharing amongst departments [ie, reallocation]. If you had your own little cash, you could keep it... [there was] no looking into, 'is what you have appropriate' [to begin with]." (\#16: Manager or other participant). "[The past approach] doesn't truly use the opportunity to be more focused on what it is you want to do as your vision for the future." (\#21: Manager or other participant, working group member). "Things have sort of grown organically .... It's not been very strategically planned" whereas PBMA is a "strategic approach." (\#11: Manager or other participant). "I think it will really take us to the essence of the question, which is 'what is our core business?" (\#13: Manager or other participant, working group member).

There were no mechanisms to get at ideas that had crosscutting impacts: "[Managers] have to look at their budget, their allocations, their individual budget lines, and they have to find cost savings. Even though they may have an idea for how work that they do interfaces with another team ... there is no mechanism to garner that kind of feedback (\#5: ELT). To focus on the core, an organization must be prepared to disinvest. The former process did not deal with this well, and PBMA could presumably do better. "Our organization is very, always was very good at identifying the new things we need to do but perhaps less good at identifying either things we don't have to do or things that we can let go of without much difference to save money." (\#18: Physician, working group member).

"We often add things on in medicine. Because the next new thing comes and we add it on, but we don't take the old thing away, right? So we get the brand new test but we keep doing the three other tests that used to be the standard. In this, it forces you to look a little bit at saying, okay, if I get this brand new piece of equipment, I'm going to stop doing this over here .... So [PBMA] articulated it not $100 \%$ but a little bit better." (\#19: Physician).

\section{B. Policy (Solution) Stream}

Three main themes emerge. Two relate to the perceived nature of the policy solution, before it was implemented: it was intuitively easy to comprehend, and it had been demonstrated elsewhere to be useful. The other theme-PBMA's ability to meet the policy problem as earlier defined-reflects informants' assessment of the process at one year after its implementation at the IWK.

Most respondents felt that PBMA was easy to understand. "Very intuitive" (\#3: ELT). "It made sense 'intuitively." (\#2: ELT). "It made sense on the face of it." (\#18: Physician, working group member). "Totally made sense." (\#11: Manager or other participant). However, there are some exceptions to this perspective. For instance, one respondent stated that PBMA "seemed somewhat complex when first introduced. It takes a while to understand what PBMA actually is in such a way that you can introduce it to others and have them understand it." (\#14: Manager or other participant).

PBMA was attractive to the IWK leaders because it had been used elsewhere, presumably successfully. This was characterized as having an 'evidence base,' a term which recurs across multiple interviews. "PBMA was chosen based on the perceived value of what PBMA had accomplished in other organizations. So it was evidence-based that we chose PBMA with respect to the literature that was out there." (\#10: Manager or other participant). "Obviously there was evidence that this had been a successful way to do things in other organizations." (\#15: Manager or other participant). The evidence was particularly associated by some with the work of one known Canadian academic proponent. His publications related to PBMA - detailing how it had been used to good effect in other organizations - were widely circulated within the IWK by project proponents prior to and during implementation. "[Name], you know, is very wellregarded, had a lot of evidence-based research around the usefulness and efficacy of that approach." (\#13: Manager or other participant, working group member).

"The process was well-founded. And evidence speaks to me. So when [Name] came and spoke to us, and I was reading his papers and looking at where this has been done in other places, that's the kind of thing that speaks to me. Where, you know, it actually has translated into something better in other places. So okay, so it's not theoretical. He's operationalized it and has proof, it works." (\#26: Manager or other participant, working group member).

Perhaps because of its reported use in several Canadian contexts, PBMA was characterized by one respondent as a "Canadian solution" to problems of resource allocation and priority setting (\#1: ELT).

It is good practice in qualitative research to look for potentially disconfirming evidence. Thus, we note here some caveats relevant to this theme, though only a small number of respondents expressed these contrary views. Some participants struggled to apply the general idea of an academically-sound process to the context of their own practice; and some expressed personal knowledge of challenges which seemed out of bounds from the more optimistic claims about evidence reported above. To address the first of these: some wanted to see evidence in practical terms, that they could apply directly to assist in implementation, and were disappointed when they perceived this not to be forthcoming. "We had asked I think on several occasions if we could have examples of how it was successful in other healthcare organizations, in particular pediatric organizations, and what sort of strategies they had put forth. But we never did get any feedback on that." (\#11: Manager or other participant). To the second point, some respondents had knowledge about PBMA from a recent, local experience (at the Dalhousie University Faculty of Medicine, also in Halifax, NS, Canada) - these participants seemed more qualified in their endorsement of it, certainly among the most cautious of those interviewed. (As noted below, however, we cannot say that these voices were representative of those who took part in the Dalhousie process. We are aware of prominent proponents who were 


\section{not interviewed.)}

PBMA was promoted in the IWK by policy entrepreneurs, who can be defined for this context as those who learned of and endorsed PBMA, brought it to the attention of their colleagues, and worked to see it put in place on a trial basis as a new means to resource allocation at the senior management level. At least three people, relatively independently, prepared arguments for adoption of PBMA. Most frequently named was a senior physician who brought information about PBMA to a member of the Executive. It is unclear how this entrepreneur learned of PBMA. Several suggestions were made by respondents - at "a conference" (\#9: Manager or other participant), from "a session or a course or something" (\#4: ELT), or from being part of the PBMA process carried out at Dalhousie Medical School, mentioned above (\#2: ELT; \#22: Manager or other participant, working group member). Another physician was also concurrently generating a case for PBMA from his own personal reading; he subsequently became a co-lead for the roll out and implementation of the initiative. Finally, arguments for PBMA as a desirable new way to approach PSRA were also being developed by an Executive Leadership Team member as part of a graduate degree program.

While these entrepreneurs became well-versed in the literature about formal methods of PSRA, the choice by the ELT and the organization as a whole to adopt PBMA was not perceived to derive from thorough rational-comprehensive comparison of all options. We asked interviewees, 'Why was the PBMA approach chosen? What other options if any were considered?' They said that, "PBMA was chosen as an alternative to our normal practices. I don't think we said there are 3 different ways we can choose from and the fourth being the way we always do things. That's not how PBMA was selected." (\#10: Manager or other participant). "It was either this or the normal way of doing business." (\#30: Manager or other participant). The normal way of doing things, according to most respondents, was across the board cuts. "The traditional cutback or the traditional control was to make a horizontal cut so everything is equal. We take a $2 \%$ or $5 \%$ or some number, what it would be, totally arbitrary." (\#3: ELT). "You could do an across the board, here's a 4\% cut to everyone, find it. So everyone gets the same cut.... That's probably the other alternative." (\#18: Physician, working group member). PBMA was deemed broadly superior to this. "The idea to move to PBMA was a good idea honestly. I don't think there was anything robust, reproducible or a realistic alternative out there, other than the standard, we just cut 5\% from everything." (\#22: Manager or other participant, working group member).

"If I compare it with other ways we have made changes in the past and tried to deal with reductions in funding or the like, basically it would be a very crude instrumenttake off your bottom $10 \%$ or bottom $5 \%$ of your staff ... or whatever. So I think this is a much wiser way to go about it." (\#2: ELT).

PBMA is also endorsed by respondents on the basis of their assessment of its impacts. Importantly, it addressed the problem as identified. First, it was seen to have increased engagement in the process. "It provided a way for people at all levels to have a voice." (\#12: Manager or other participant).
"It was a good approach to getting frontline staff engagement and involvement" (\#27: Manager or other participant). This broad engagement appeared to produce a number of the benefits which had been expected:

"You had the organization, and when I say the organization I mean everybody within the organization, involved in the process rather than having an Executive Leadership or somebody else in some sort of isolated room making decisions about where funding was going to be cut. And so it was allowing that participation. And I think that that in and of itself made the process credible." (\#20: Manager or other participant).

Second, PBMA - according to respondents - made it harder for decision-maker s to advocate solely for their own constituency or silo. "What PBMA did that our current business practices perhaps did not do as well is to encourage folks to look over those silos." (\#10: Manager or other participant). "It gave us a lens of what's happening across the institution through which to make resource allocation decisions. Which I think is better than doing it in isolation and silos." (\#26: Manager or other participant, working group member). "From a hospital point of view rather than being siloed and people talking individually within each individual department, this was a hospital wide process. There were representatives from all the different areas." (\#20: Manager or other participant). There was "a need for people to really try to understand sort of what they could do internally and then how that could be shifted so that the whole organization benefits. So that was a shift, instead of looking at your own isolated program area, to think more broadly." (\#30: Manager or other participant).

\section{Politics Stream}

Canadian healthcare organizations like the IWK, being publicly funded, are constantly aware that they are subject to the demands of provincial Ministries of Health. The main theme from this case study related to the politics stream is that, despite a certain degree of provincial unhappiness, the IWK was allowed to pursue its preferred solution to the defined problem - though some limits were imposed as to politically acceptable outcomes.

The CEO is the primary interface between the organization and the province.

"My main role is to listen very closely to the people that I get advice from - my vice-presidents and others. And then to put that together with what I know is happening outside and around us politically and all of that to make sure that what we think will work for us fits within the context of what the province is trying to do."

The CEO's willingness (and that of the senior management as a collective) to stand up for PBMA in the face of pressures from above was remarked upon. "Despite the fact that there ... was pressure from the government, I think [CEO] was a tireless champion." (\#22: Manager or other participant, working group member). Provincial interest in shaping the IWK's resource allocation process was evident both before and during the PBMA exercise. "The government hear[d] about PBMA and stepp[ed] in, saying, 'Can you give us a list of your quick wins'? Which we said, no, we're going to follow the process the whole way through.” (\#22: Manager or other participant, working group member). Others described a 
need here for the IWK leaders to be cautious:

"You don't want to, kind of, say 'well if we have to cut here's how we're going to do it'. That becomes almost an invitation for somebody in the government to say, 'oh, look, they've got plans about where we can take money and cut'. So it is a bit of a fine line. You want to have some ideas yourself but you don't necessarily want to be making that public. It's just that you need to be kind of on track with your planning.” (\#21: Manager or other participant, working group member).

Several respondents suggested that their preferred option would have been to see PBMA enacted at a province-wide level. That window was not open. "We tried to encourage the entire province to do it, you know, through Department of Health. They were looking at it, but it is a tremendous amount of work and it's political when you get to that level." (\#5: ELT).

"We thought initially that it would be an excellent provincial initiative, and we tried to encourage that. We talked to the province, to the bureaucrats and probably some of the elected people, I can't remember, and I think although they thought it was a good idea, it seemed to be a daunting task, and it was going to cost money." (\#1:ELT). While the IWK received the leeway it needed to proceed with PBMA, nonetheless, politics impinged on the process through the province's 'veto' of certain disinvestment choices. Respondents expressed this in both general and specific terms. The "frustration is if we think it's not important but the government says you're not allowed to cut there, okay, well who would know better than us?" (\#18: Physician, working group member).

"The government of the day was a very labour-focused government. And as a result, any PBMA that was approved by the working group that had an HR impact at all was not allowed to go forward. So some great ideas came forward that we weren't allowed to move on even though they were approved" (\#5: ELT).

The convergence of these 3 streams, opening a policy window for the introduction of PBMA at the IWK, is illustrated in Figure.

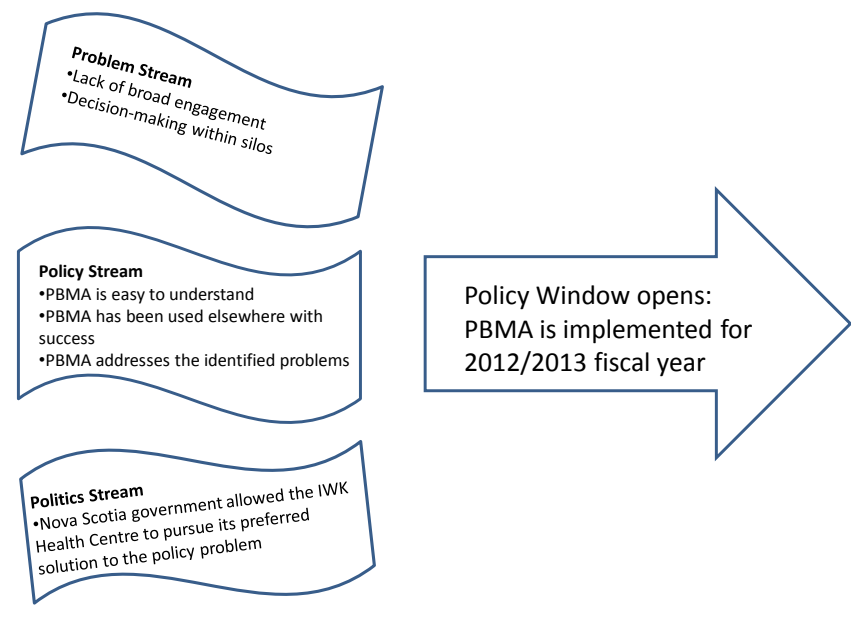

Figure. Problem, Policy and Politics Streams Converge in the Opening of a Policy Window for Implementation of PBMA at the IWK Health Centre. Abbreviation: PBMA, Program Budgeting and Marginal Analysis.
Discussion and Conclusion

This study reports one case where a policy window opened; ie, it was possible to introduce meaningful change to PSRA practice at the IWK. Policy entrepreneurs pitched an acceptable solution to an agreed problem. That problem was defined as lack of broad engagement and silo'd decisionmaking. Despite the substantial financial pressures facing the organization, the PSRA problem was NOT seen primarily as the need for a mechanism to reach budget targets or about having a more precise technical formula for calculating costs and benefits. PBMA linked the problem and policy streams while also conforming to political expectations as well as constraints. Despite hesitation, the province allowed the IWK to proceed with PBMA. Being able to argue that resource allocation decisions were reached in an explicit, formal, evidence-based process gave management at the IWK some way to shield (at least partly) their choices from provincial intervention, in line with arguments made elsewhere in the literature. $^{2}$

Generally, respondents reported that the expected benefits were obtained. We have noted throughout some of the contrasting minority claims. Participants showed no demonstrable reluctance to criticize organizational performance where they felt it warranted; individual interviews with a perceived neutral party may have enabled this in a way that focus groups would not. Qualitative research does not judge the weight of evidence by strictly totalling the number of times an idea is expressed-even a response that comes from only one individual can be important if it points to aspects of the question that provide greater understanding and which flag areas for improvement. In this sense, the fact that we could not interview all of the suggested informants is a potential limitation to the findings, if any of them held a significantly different or unique view that we could not otherwise access. ${ }^{20}$

However, the larger literature does seem to confirm that PBMA in other contexts has proven able to achieve the benefits for which it was sought here. Gibson, Martin, and Singer have argued that a condition for fair process is 'empowerment'that is, that process design should "optimise opportunities for effective participation." ${ }^{21}$ This has been operationalized in priority setting interventions. ${ }^{22,23}$ PBMA studies have also previously reported that vertical budget silos and lack of integration pose significant barriers to effective resource allocation..$^{24}$ Some PBMA exercises have reported success in breaking down departmental walls. In one Canadian case, senior decision-makers felt that the process had produced, "greater consideration of the linkages between departments and portfolios"25; in the United Kingdom, a PBMA effort appeared to encourage "more joined up working." ${ }^{26}$ Literature also argues the need for clinical champions ${ }^{27,28}$; their presence in this case was soundly demonstrated both among initial policy entrepreneurs as well as among co-leads for the project implementation.

Policy-makers often find that studies which employ models, such as Kingdon's version of agenda setting theory, in their analysis fail to offer pragmatic or actionable suggestions. ${ }^{29}$ We attempt therefore to draw out some of the implications of this case study for PSRA practice. Multiple streams theory suggests that change is only possible when the streams merge, 
which is a rare occurrence. There will be many times then when the situation is not right, where the barriers outweigh the enablers, and it is not worth the effort to pursue formal priority setting. ${ }^{24}$ Understanding and perhaps assessing organizational readiness ${ }^{30}$ may be a useful strategy that could be employed by the potential policy entrepreneur. Decision-makers particularly should think through whether the proposed solution fits with the definition of the problem. Multiple streams theory contends that proponents try to attach their preferred policy to any seemingly promising opportunity. In the IWK it worked - PBMA proved to possess qualities that addressed the agreed deficiencies in priority setting. But there will surely be cases where PBMA or other formal models do not fit the problem or political imperatives of the day. In other words, an academically sound approach might not always fit in any given context.

In a general sense, the multiple streams concepts of problem, policy and political streams have been useful in a wide range of studies. A recent comprehensive review found reports from 65 countries; while $78 \%$ of these were Western liberal democracies, Oceania, Asia, Africa, and Latin America are all represented. ${ }^{31}$ We have demonstrated here that multiple streams theory is useful to understand PSRA in the health sector; a similar lens could be illuminating of change efforts across a broad variety of national contexts. Of course, using a template such as multiple streams is based on a presumption by the analysts that research data will fit within the categories given. Valuable complementary or alternative insights can be gained by employing other qualitative approaches. ${ }^{32}$ Our research team has prepared other analyses of the evaluative data arising from the IWK experiences; these will be available elsewhere and can be obtained from the authors on request. PBMA at the IWK was posited as an alternative to business as usual but was not itself chosen via a rational-comprehensive comparison of all options. In other words, to be adopted it needs to be seen as better than current practice, but not necessarily as unequivocally the one best model. This demonstrates the concept of satisficing. ${ }^{33}$ Respondents here with more direct previous experience of PBMA through its use at the Dalhousie University Faculty of Medicine were tempered in their enthusiasm; this may not be representative, as the majority of all participants in that project "were very clear that they preferred the PBMA process to across-the-board spending cuts." ${ }^{34}$ That appears to be the same dichotomous choice that managers at the IWK felt confronted them in their priority setting practice (aside perhaps from the initial policy entrepreneurs who more deeply investigated the question). Senior Canadian healthcare decision-makers overall report better experience with formal processes, whatever those may be, than with more politically or historically-grounded options. ${ }^{35}$

Notwithstanding its international origins, ${ }^{36}$ much of the recently published work with PBMA occurs in Canadian contexts. $^{23,30,37-40}$ To an extent, this might make it appear to Canadians as a 'Canadian' solution. Nonetheless, the findings should have some general interest within the international healthcare management community in terms of potentially transferrable lessons. What conditions are likely to be similar? Many healthcare organizations, in developed and developing countries, have well-regarded and reputable local champions who can push for changes that make better use of scarce resources. Being perceived as an intuitive or easy-tounderstand approach can also help facilitate process adoption - this is a factor likely to be of universal appeal. Getting consensus on re-allocation through inclusive, bottom-up initiatives that generate buy-in among key actors is also a goal likely to be transferrable to many situations. Proposed policy changes should match the goals they are meant to achieve. That said, as far as the IWK's chosen model of priority setting, "PBMA and extensions of the approach have been applied and evaluated primarily in high-income countries. Their application to a low-resource setting has yet to be documented. In these settings an additional number of systemic, political and otherwise context-specific issues exist. These issues will impact on the extent to which an evidence-based process as proposed by PBMA is needed, feasible and likely to achieve actual improvements to resource allocation." ${ }^{41}$

Further research can help elaborate the channels by which such knowledge and information flows among decision-makers. The suggestion here that knowledge-as a combination of literature and what is promoted via conferences and other forms of peer-to-peer exchange is taken up and spread by entrepreneurs, advocates and healthcare leaders, is consistent with other recent Canadian research looking at how organizations are identified by peers as 'high performers' in PSRA. ${ }^{42}$ The larger literature around knowledge translation and exchange (KTE) identifies several types of KTE process. The situation at the IWK perhaps most closely resembles the 'user pull' model: decision-makers seek out knowledge which might help them to solve an identified program confronting their organization. ${ }^{43}$ In addition to searching scientific literature independently or in a postsecondary setting, decision-makers can seek knowledge through networks or communities of practice. ${ }^{44}$ These can arise spontaneously, or be deliberately constructed as venues for sharing practice-relevant knowledge. In Canada, we know of no examples of the second sort specific to PSRA. In policy studies, concepts of policy diffusion similarly look at the spread of knowledge as seen in the uptake of policy or program choices ${ }^{45}$; policy diffusion literature tends to consider large-scale patterns, compared to the more finegrain qualitative understanding of how actors in one place actually find information and the issues they encounter when transporting it into their unique organizational context. To our knowledge, no such study of the spread of PSRA practices has been undertaken.

In conclusion, meaningful change is not brought about simply because health providers 'need to cut' budgets (though that is an important part of the political and organization context on many occasions). This study shows that there are other factors, revolving largely around perceptions about the nature of priority setting problems and the feasible policy solutions to these. The importance of both management and clinical leadership in advocating for and steering practice change has previously been noted; our study confirms this. Using the concept of the policy entrepreneur allows us to get additional purchase on how such roles might play out in particular settings. Satisficing applies: Healthcare decision-makers typically agree that formal approaches are better than purely 
historical or politically-driven ones. Looking at what has been done elsewhere can identify options. PBMA has been utilized in a wide range of different contexts. Where careful consideration shows it might fit the nature of the problem and political context, many healthcare organizations have found it a valuable means to improve resource allocation and increase the benefits obtained from limited funds.

\section{Acknowledgements}

The authors thank Dr. Evelyn Cornelissen for her helpful comments on early drafts of this manuscript. This research was funded by the Canadian Institutes of Health Research, through the Evidence Informed Health Care Renewal (Policy Analysis) stream. The IWK Health Centre provided in-kind support for local data collection.

\section{Ethical issues}

The ideas expressed in this paper should be considered the personal opinions of the authors and not necessarily the view of the IWK Health Centre, Halifax, NS, Canada.

\section{Competing interests}

Authors declare that they have no competing interests.

\section{Author' Contributions}

MAH and MC, with the assistance of $\mathrm{CM}$, carried out the original project on which this research is based. MAH and CM conceived the research project and served as co-principal investigators; MC, SAG, and NS developed the research protocol and funding proposal. LD, with the support of NS, and under the supervision of MC and SAG, conducted the data collection. LD and NS conducted the initial data analysis, with results reviewed by and discussed with all members of the research team. NS prepared the initial draft of the manuscript; all authors reviewed, provided feedback on, and approved the final manuscript.

\section{Authors' affiliations}

${ }^{1}$ Centre for Clinical Epidemiology \& Evaluation, Vancouver Coastal Health Research Institute, University of British Columbia, Vancouver, BC, Canada. ${ }^{2} S$ chool of Population and Public Health, University of British Columbia, Vancouver, BC, Canada. ${ }^{3}$ Dalhousie University, Halifax, NS, Canada. ${ }^{4}$ Strategy and Organizational Performance, IWK Health Centre, Halifax, NS, Canada. ${ }^{5}$ Faculty of Medicine, Dalhousie University, Halifax, NS, Canada.

\section{References}

1. Barasa E, Molyneux WS, English M, Cleary S. Setting healthcare priorities in hospitals: a review of empirical studies. Health Policy Plan. 2015;30(3):386-396. doi:10.1093/heapol/czu010

2. Smith N, Mitton C, Davidson A, Williams I. A politics of priority setting: ideas, interests and institutions in healthcare resource allocation. Public Policy Adm. 2014;29(4):331-347. doi:10.1177/0952076714529141

3. Sabatier P. The need for better theories. In: Sabatier PA, ed. Theories of the Policy Process . 2nd ed. Westview: Boulder, CO; 2007:3-17.

4. Breton E, De Leeuw E. Theories of the policy process in health promotion research: a review. Health Promot Int. 2010;26(1):8290. doi:10.1093/heapro/daq051

5. Kingdon JW. Agendas, Alternatives, and Public Policies. 2nd ed. New York, NY: Addison-Wesley Educational Publishers Inc; 1995.

6. Nowlin MC. Theories of the policy process: state of the research and emerging trends. Policy Stud J. 2011;39(S1):41-60. doi:10.1111/j.1541-0072.2010.00389_4.x

7. Ridde V. Policy implementation in an African state: an extension of Kingdon's multiple-streams approach. Public Adm. 2009;87(4):938-954. doi:10.1111/j.1467-9299.2009.01792.x
8. Gulbrandsson K, Fossum B. An explanation of the theoretical concepts policy windows and policy entrepreneurs at the Swedish public health arena. Health Promot Int. 2009; 24(4):434444. doi:10.1093/heapro/dap033

9. Vos B, Lagasse R, Levesque A. Putting newborn hearing screening on the political agenda in Belgium: local initiatives toward a community programme - a qualitative study. Health Res Policy Syst. 2014;12:32. doi:10.1186/1478-4505-12-32

10. Blackman VS. Putting policy theory to work: tobacco control in California. Policy Polit Nurs Pract. 2005;6(2):148-155. doi:10.1177/1527154405276289

11. Craig RL, Felix HC, Walker JF, Phillips MM. Public health professionals as policy entrepreneurs: Arkansas's childhood obesity policy experience. Am J Public Health. 2010;100(11):20472052. doi:10.2105/ajph.2009.183939

12. Gladwin C, Church J, Plotnikoff R. Public policy processes and getting physical activity into Alberta's urban schools. Can J Public Health. 2008;99(4):32-38.

13. Henstra D. Explaining local policy choices: a multiple streams analysis of municipal emergency management. Can Public Adm. 2010;53(2):241-258. doi:10.1111/j.1754-7121.2010.00128.x

14. Rowlands $\mathrm{IH}$. The development of renewable electricity policy in the province of Ontario: the influence of ideas and timing. Rev Policy Res. 2007;24(3):185-207. doi:10.1111/j.15411338.2007.00277.x

15. Mannheimer LN, Gulis G, Lehto J, Ostlin P. Introducing health impact assessment: an analysis of political and administrative intersectoral working methods. Eur $J$ Public Health. 2007;17(5):526-531. doi:10.1093/eurpub/ckl267

16. Tenbensel T. Complexity theory and health policy. In: Geyer R, Cairney P, eds. Handbook on Complexity and Public Policy. Edward Elgar Publishing; 2015.

17. Peacock S, Mitton C, Ruta D, Donaldson C, Bate A, Hedden L. Priority setting in healthcare: towards guidelines for the program budgeting and marginal analysis framework. Expert Rev Pharmacoeconomics Outcomes Res. 2010;10(5):539-552. doi:10.1586/erp.10.66

18. Yin R. Case Study Research: Design and Methods. 4th ed. Thousand Oaks, CA: Sage; 2009.

19. King N. Using templates in the thematic analysis of text. In: Cassell C, Symon G, eds. Essential Guide to Qualitative Methods in Organizational Research,. London: Sage; 2004.

20. Groger L, Mayberry PS, Straker JK. What we didn't learn because of who would not talk to us. Qual Health Res. 1999;9(6):829-835. doi:10.1177/104973299129122180

21. Gibson JL, Martin DK, Singer PA. Priority setting in hospitals: fairness, inclusiveness, and the problem of institutional power differences. Soc Sci Med. 2005;61:2355-2362. doi:10.1016/j. socscimed.2005.04.037

22. Gibson JL, Mitton C, DuBois-Wing G. Priority setting in Ontario's LHINs: ethics and economics in action. Healthcare $Q$. 2011;4(4):35-43. doi:10.12927/hcq.2011.22649

23. Urquhart $B$, Mitton $C$, Peacock $S$. Introducing priority setting and resource allocation in home and community care programs. $J$ Health Serv Res Policy. 2008;13(suppl 1):41-45. doi:10.1258/ jhsrp.2007.007064

24. Mitton C, Donaldson C. Setting priorities and allocating resources in health regions: lessons from a project evaluating Program Budgeting and Marginal Analysis. Health Policy. 2003;64:335348. doi:10.1016/s0168-8510(02)00198-7

25. Mitton C, Patten S, Donaldson C. Listening to the decision makers: sustainability of PBMA in Alberta. Appl Health Econ Health Policy. 2004;3(3):143-151. doi:10.2165/00148365200403030-00005

26. Goodwin E, Frew EJ. Using programme budgeting and marginal 
analysis (PBMA) to set priorities: reflections from a qualitative assessment in an English Primary Care Trust. Soc Sci Med. 2013; 98:162-168. doi:10.1016/j.socscimed.2013.09.020

27. Harrison $A$, Mitton $C$. Engaging physicians in priority setting: the Alberta experience. Healthc Manage Forum. 2004;17(4):21-27.

28. Ruta D, Mitton C, Bate A, Donaldson C. Programme Budgeting and Marginal Analysis: bridging the divide between doctors and managers. BMJ. 2005;330(7506):1501-1503. doi:10.1136/ bmj.330.7506.1501

29. Lomas J, Brown A. Research and advice giving: a functional view of evidence-informed policy advice in a Canadian ministry of health. Milbank Q. 2009;87:903-926.

30. Cornelissen E, Mitton C, Davidson A, et al. Changing priority setting practice: the role of implementation in practice change. Health Policy. 2014;117(2):266-274. doi:10.1016/j. healthpol.2014.04.010

31. Jones MD, Peterson $\mathrm{HL}$, Pierce JJ, et al. A river runs through it: a multiple streams meta-review. Policy Stud J. 2015. doi:10.1111/ psj.12115

32. Burck C. Comparing qualitative research methodologies for systemic research: the use of grounded theory, discourse analysis and narrative analysis. J Fam Ther. 2005;27:237-262. doi:10.1111/j.1467-6427.2005.00314.x

33. March JG, Simon HA. Organizations. New York: John Wiley and Sons; 1958.

34. Mitton C, Levy A, Gorsky D, MacNeil C, Dionne F, Marrie T. Allocating limited resources in a time of fiscal constraints: a priority setting case study from Dalhousie University Faculty of Medicine. Acad Med. 2013;88(7):939-945. doi:10.1097/ acm.0b013e318294fb7e

35. Smith N, Mitton C, Bryan S, et al. Decision maker perceptions of resource allocation processes in Canadian health care organizations: a national survey. BMC Health Serv Res. 2103;13:247. doi:10.1186/1472-6963-13-247

36. Madden L, Hussey R, Mooney G, Church E. Public health and economics in tandem: Programme budgeting, marginal analysis and priority setting in practice. Health Policy. 1995;33(2):161-
168. doi:10.1016/0168-8510(95)93676-r

37. Dionne F, Mitton C, Smith N, Donaldson C. Evaluation of the impact of Program Budgeting and Marginal Analysis in Vancouver Island Health Authority. J Health Serv Res Policy. 2009;14(4):234-242. doi:10.1258/jhsrp.2009.008182

38. Mitton C, Mackenzie J, Cranston L, Teng F. Priority setting in the Provincial Health Services Authority - case study for the 2005/2006 planning cycle. Healthc Policy. 2006;2(1):91-106.

39. Mitton C, Dionne F, Damji R, Campbell D, Bryan S. Difficult decisions in times of constraint: criteria based resource allocation in the Vancouver Coastal Health Authority. BMC Health Serv Res. 2011;11:169. doi:10.1186/1472-6963-11-169

40. Patten S, Mitton C, Donaldson C. Using participatory action research to build a priority setting process in a Canadian regional health authority. Soc Sci Med. 2003;63:1121-1134. doi:10.1016/j. socscimed.2006.01.033

41. Jimenez-Soto E, Alderman K, Hipgrave D, Firth S, Anderson A. Prioritization of investments in reproductive, women's and children's health: evidence-based recommendations for low and middle income countries in Asia and the Pacific - a subnational focus. http://www.who.int/pmnch/activities/advocacy/20121028_ prioritise_investments.pdf. Published 2012.

42. Smith N, Hall W, Mitton C, Bryan S, Urquhart B. What constitutes high performance in priority setting and resource allocation? Decision maker narratives identified from a survey and qualitative study in Canadian healthcare organizations. Health Serv Manage Res. 2014 Dec 4. pii: 0951484814559714.

43. Lavis JN. Research, public policymaking, and knowledgetranslation processes: Canadian efforts to build bridges. J Contin Educ Health Prof. 2006;26(1):37-45.

44. Barwick MA, Peters J, Boydell K. Getting to uptake: do communities of practice support the implementation of evidence-based practice? J Can Acad Child Adolescent Psych. 2009;18(1):16-29.

45. Shipan CR, Volden C. Policy diffusion: seven lessons for scholars and practitioners. Public Adm Rev. 2012;72(6):788796. doi:10.1111/j.1540-6210.2012.02610.x 